\title{
Age-Period-Cohort Analysis of Type-Specific Stroke Morbidity and Mortality in China
}

\author{
Yuan Wang, PhD; Qin Peng; Jian Guo; Lihui Zhou; Wenli Lu, PhD
}

\begin{abstract}
Background: Stroke has become the leading cause of death in China. This study aimed to assess the age-period-cohort (APC) effects on the long-term trends of type-specific stroke morbidity and mortality in China between 1993 and 2017.
\end{abstract}

\begin{abstract}
Methods and Results: The data were obtained from the Global Burden of Disease 2017 (GBD 2017) and were analyzed with the age-period-cohort framework. The net drifts of mortality were below 0 (hemorrhagic stroke [HS]: males: $-1.620 \%$, females: $-3.531 \%$; ischemic stroke [IS]: males: $-1.041 \%$, females: $-3.002 \%)$, and the local drift values were below 0 in all age groups and for both genders. The net drifts of HS incidence were below 0 (males: $-1.412 \%$, females: $-2.688 \%$ ), while those of IS were above 0 (males: $1.425 \%$, females: $1.117 \%$ ). Period effect of mortality showed similar monotonic downward patterns for both genders, with a faster decrease for females than for males. Period effect of incidence showed a declined trend of incidence for HS, but an elevated trend for IS in both genders. After controlling for age and period effects, cohort effects on incidence found a monotonic decline trend for HS, while for IS, an elevated trend was found at first to peak during the 1950-1970s, then declined steadily afterwards. Cohort effects on mortality showed a monotonic declined trend.
\end{abstract}

Conclusions: By using Age-Period-Cohort (APC) analysis, a disparity between HS and IS was identified. Different prevention and control strategies should be used depending on the subtypes of stroke.

Key Words: Age-period-cohort analysis; Hemorrhagic stroke; Incidence; Ischemic stroke; Mortality

$\mathbf{S}$ troke has become the leading cause of mortality globally and in China. During the past 3 decades, the risk of stroke in China has increased rapidly due to health transitions and sociodemographic changes. From 1990 to 2016, stroke occurrences had more than doubled to $\sim 5,510,276$, and the stroke mortality had increased by $29.06 \% .^{1}$ Stroke incidence and mortality rate increases with aging. The age-standardized incidence of ischemic stroke (IS) increased dramatically, but that of hemorrhagic stroke (HS) decreased slightly, ${ }^{2}$ and the age-standardized mortality caused by stroke decreased dramatically. ${ }^{3}$

To better understand the long-term trend of stroke burden, an age-period-cohort (APC) model has been used to investigate the possible reasons underlying the temporal trend. The APC model is a comprehensive method that considers the effect of age, period and birth cohort. The majority of APC studies of stroke mainly focused on mortality. ${ }^{48}$ Yet, other important factors such as age, period, birth cohort effect on type-specific stroke incidence and mortality has not been fully investigated.

We aimed to investigate the long-term trends of typespecific stroke incidence and mortality in China between 1990 and 2017, examining age-, period-, and cohort-specific effects by gender using the APC framework. We used the data from the Global Burden of Disease Study 2017 (GBD 2017). In doing so, we anticipate that findings from our study could provide etiologic implications for the Chinese population, and provide evidence-based information to inform prevention strategies.

\section{Methods}

\section{Data Sources}

This study used the data from the GBD 2017. The GBD study used DisMod-MR 2.1, a Bayesian meta-regression tool, as the main method of estimation, ensuring consistency between rates of incidence, prevalence, remission, and cause of death for each condition. The GBD study provides internally consistent estimates of age- and gender-specific all-cause and cause-specific mortality for 282 causes of death and incidence for 354 diseases and injuries globally, regionally, and nationally from 1990 to 2017. Stroke was identified based on the 9th and 10th revision of the International Classification of Disease. In the GBD data set, stroke incidence and mortality were diagnosed and were defined based on the World Health Organization clinical

Received September 20, 2019; revised manuscript received January 10, 2020; accepted February 4, 2020; J-STAGE Advance Publication released online March 10, 2020 Time for primary review: 32 days

Department of Epidemiology and Biostatistics, School of Public Health, Tianjin Medical University, Tianjin (Y.W., Q.P., L.Z., W.L.); Tianjin Bin Hai New Area Center for Diseases Control and Prevention, Department of STD\&AIDS Control and Prevention, Tianjin (J.G.), China

Mailing address: Wenli Lu, PhD, Department of Epidemiology and Biostatistics, School of Public Health, Tianjin Medical University, No. 22 Qi Xiang Tai Road, He Ping District, Tianjin 300070, China. E-mail: luwenli@tmu.edu.cn

ISSN-1346-9843 All rights are reserved to the Japanese Circulation Society. For permissions, please e-mail: cj@j-circ.or.jp 
A

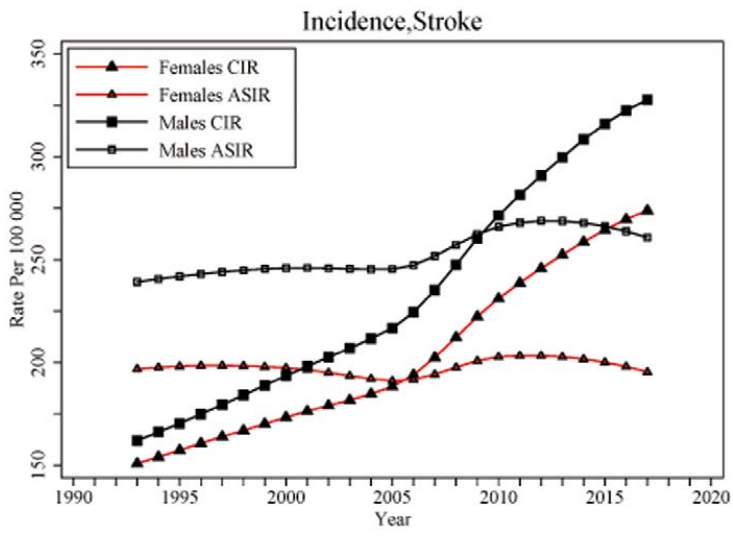

B

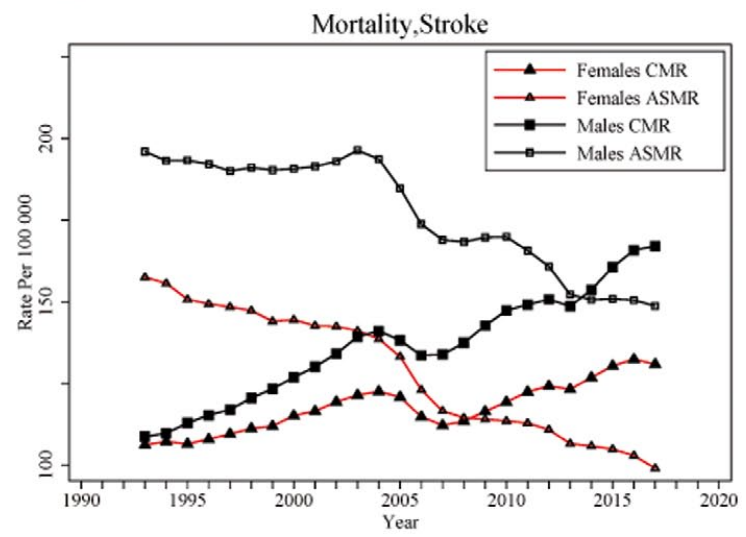

Figure 1. Trends of the crude incidence/mortality rates and the age-standardized incidence/mortality rates per 100,000 population for stroke by gender in China during 1993 to 2017. (A) Trends of the crude incidence rates (CIR) and the age-standardized incidence rate (ASIR). (B) Trends of the crude mortality rates (CMR) and the age-standardized mortality rate (ASMR).

criteria and the International Statistical Classification of Diseases, Ninth (ICD-9) and Tenth (ICD-10) revisions and Related Health Problems. ${ }^{9}$ Stroke mortality was defined using ICD-9 codes (430-435.9, 437.0-437.2, 437.5-437.8) and ICD-10 codes (G45-G46.8, I60-I63.9, I65-I66.9, I67.0 I67.3, I67.5-I67.6, I68.1-I68.2, I69.0-I69.3). IS mortality was defined using ICD-9 codes (433-435.9, 437.0-437.1, 437.5-437.8) and ICD-10 codes (G45-G46.8, I63-I63.9, I65-I66.9, I67.2-I67.3, I67.5-I67.6, I69.3). HS mortality was defined using ICD-9 codes $(431-432.9,437.2)$ and ICD10 codes (I61-I62, I62.1-I62.9, I68.1-I68.2, I69.1-I69.2).

To characterize the temporal trends in China, stroke incidence and mortality rates for males and females were age-standardized by using the GBD 2017 data for a global age-standard population. ${ }^{10}$

\section{APC Model}

The standard APC model assumed that the observed number of diseases followed a Poisson distribution and that the incidence or mortality was a multiplicative function of age, cohort and period, such that the logarithm of the rates is an additive function of the parameters. ${ }^{11-14}$ With the aim of detailed analyses, we performed an APC analysis using the R-based web tool from the US National Cancer Institute (http://analysistools.nci.nih.gov/apc/). ${ }^{15}$ The benefit of this online web tool was that the output was easy to understand and it can solve the problem of identifying model parameters such as the exact linear dependence of the regression variables (Age $=$ Period - Cohort $)$ causing the identifiable problem.

The age effects represented differing risks of the outcomes associated with different age brackets; the period effects represented variations in the outcomes over time that influenced all age groups simultaneously; the cohort effects were associated with the changes of outcomes across groups of individuals with the same birth years. Holford has proposed that if age, period, and cohort trends were orthogonally decomposed into their linear and non-linear parts, many useful functions can be estimated. ${ }^{16}$

In this study, we mainly focused on the following estimable functions: ${ }^{15}$ net drift, which was an analog of the annual percent change in the incidence and mortality over the study period, and it estimated the average annual percentage change in the logarithm of the incidence and mortality with the adjustments of a non-linear period and cohort effects; local drift indicated the average annual percentage changes in incidences over time across different age groups; longitudinal age curve indicated the fitted longitudinal age-specific rates in the reference cohort adjusted for period deviations; the period (or cohort) rate ratios (RR) would be the relative risk adjusted for age and non-linear effects in a period (or cohort) vs. the reference. To conduct APC analysis, the mortality and population data were arranged into consecutive 5-year periods from 1993 to 2017 and successive 5-year age intervals from 15 years to $85 \sim$ years. The central age group, period, and birth cohort were defined as the reference in all APC analyses; in case of an even number of categories, the reference value was set as the lower of the 2 central values. Wald chi-squared tests were adopted for the significance of the estimable functions. To compare the significance of the slope of the period/cohort RRs, we used a general linear model to check the interaction effect between gender and calendar year/birth cohort. All statistical tests were 2-sided.

\section{Results}

\section{Incidence and Mortality Trend of Stroke}

Trends of the crude incidence/mortality rates (CIR/CMR) and the age-standardized incidence rates (ASIR)/mortality rates (ASMR) for stroke by gender for the period of 1993 to 2017 are shown in Figure 1. The CIR for stroke showed overall increasing trends, from 161.9 to 327.8 per 100,000 for males and 150.9 to 274.0 per 100,000 for females. The CMR in both genders also showed increasing trends, from 108.8 to 167.1 per 100,000 for males and 106.2 to 130.9 per 100,000 for females. After age-standardization, the ASIR showed a slightly increasing trend; the ASMR showed a decreasing trend for both males and females.

\section{APC Analysis}

Age group-specific annual percent changes (local drifts) with 

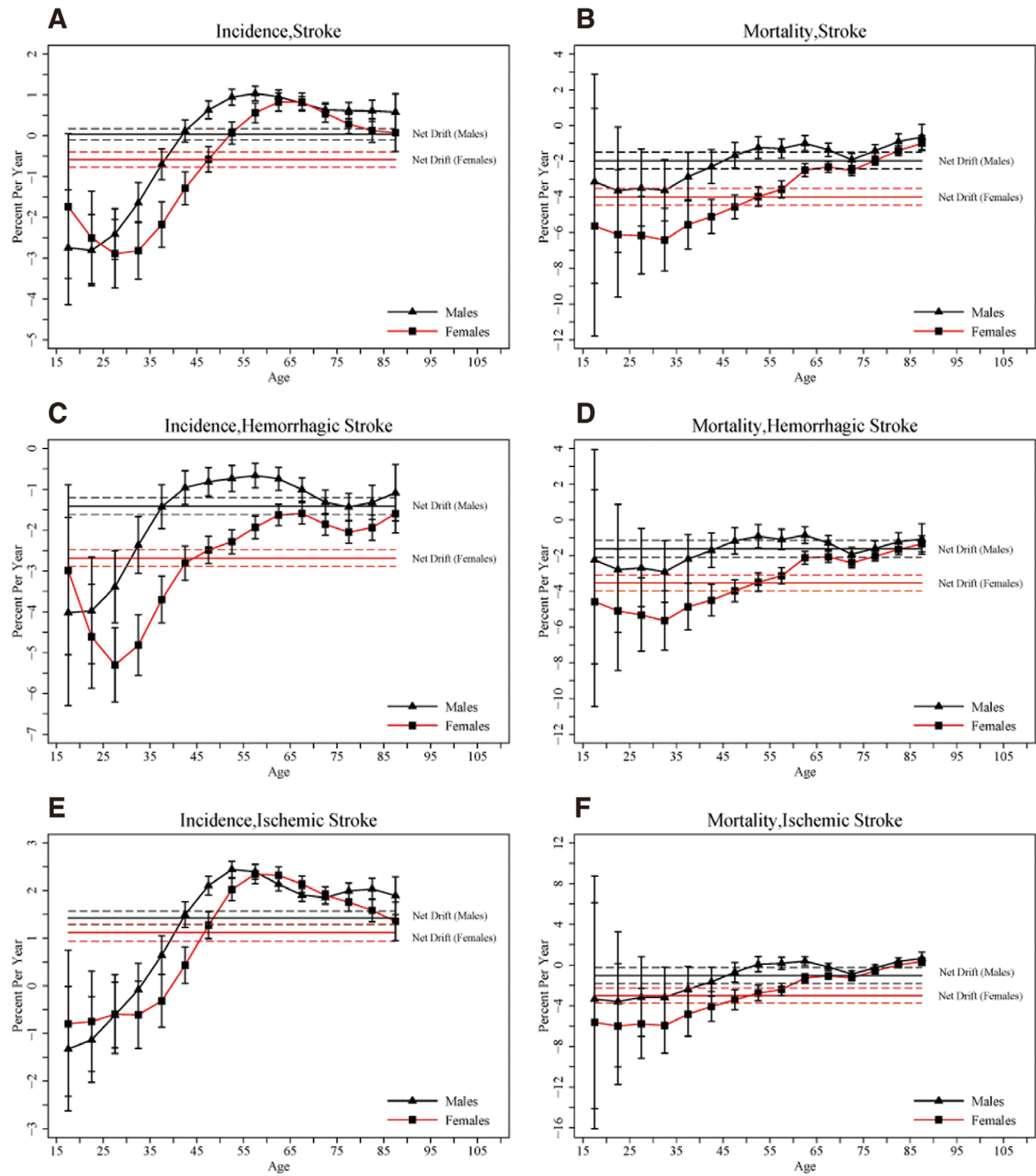

Figure 2. Age group-specific annual percent change (local drift) with the overall annual percent change (net drift) for type-specific stroke incidence/mortality rates with 95\% confidence intervals. (A) Local drift with net drift for Stroke incidence rate. (B) Local drift with net drift for Stroke mortality rate. (C) Local drift with net drift for HS incidence rate. (D) Local drift with net drift for HS mortality rate. (E) Local drift with net drift for IS incidence rate. (F) Local drift with net drift for IS mortality rate.

the overall annual percent change (net drift) in stroke incidence/mortality for different genders are shown in Figure 2. We found that the majority net drift values were below 0 (Figure 2, Table), the exceptions being the net drift values for males in stroke incidence $(0.037 \%[95 \%$ $\mathrm{CI}=-0.100,0.175]$ per year), for males in IS incidence $(1.425 \%[95 \% \mathrm{CI}=1.284,1.566]$ per year) and for females in IS mortality $(1.117 \%$ [95\% $\mathrm{CI}=0.936,1.298]$ per year $)$.

The local drift values for stroke incidence reached its maximum at age $\sim 60$ years in males (Figure 2A). All of the HS-related local drift values were below 0 in all age groups in both genders (Figure 2C). For IS, the local drift values were almost above 0 after age 35 years for males and after age 40 years for females (Figure 2E). However, regarding 


\begin{tabular}{|c|c|c|c|c|c|}
\hline \multirow[b]{2}{*}{ Disease / Sex } & \multirow[b]{2}{*}{ Type } & \multirow{2}{*}{$\begin{array}{c}\text { Net drift } \\
(\% \text { per year; } 95 \% \mathrm{Cl})\end{array}$} & \multicolumn{3}{|c|}{$P$ value } \\
\hline & & & $\begin{array}{c}\text { All local } \\
\text { drifts=net drift }\end{array}$ & $\begin{array}{c}\text { All cohort } \\
\text { deviations }=0\end{array}$ & $\begin{array}{c}\text { All period } \\
\text { deviations }=0\end{array}$ \\
\hline \multicolumn{6}{|l|}{ Stroke } \\
\hline \multirow[t]{2}{*}{ Male } & Incidence & $0.037(-0.100,0.175)$ & $<0.001$ & $<0.001$ & $<0.001$ \\
\hline & Mortality & $-1.965(-2.428,-1.500)$ & 0.001 & $<0.001$ & 0.514 \\
\hline \multirow[t]{2}{*}{ Female } & Incidence & $-0.584(-0.774,-0.394)$ & $<0.001$ & $<0.001$ & $<0.001$ \\
\hline & Mortality & $-3.995(-4.461,-3.526)$ & $<0.001$ & $<0.001$ & 0.009 \\
\hline \multicolumn{6}{|c|}{ 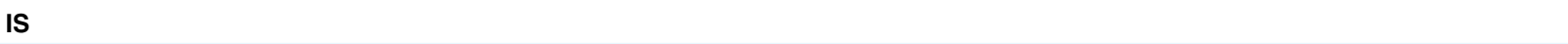 } \\
\hline \multirow[t]{2}{*}{ Male } & Incidence & $1.425(1.284,1.566)$ & $<0.001$ & $<0.001$ & $<0.001$ \\
\hline & Mortality & $-1.041(-1.824,-0.252)$ & $<0.001$ & $<0.001$ & 0.411 \\
\hline \multirow[t]{2}{*}{ Female } & Incidence & $1.117(0.936,1.298)$ & $<0.001$ & $<0.001$ & $<0.001$ \\
\hline & Mortality & $-3.002(-3.747,-2.251)$ & $<0.001$ & $<0.001$ & 0.042 \\
\hline \multicolumn{6}{|l|}{ HS } \\
\hline \multirow[t]{2}{*}{ Male } & Incidence & $-1.412(-1.619,-1.205)$ & $<0.001$ & $<0.001$ & $<0.001$ \\
\hline & Mortality & $-1.620(-2.093,-1.144)$ & 0.015 & 0.031 & $<0.001$ \\
\hline \multirow[t]{2}{*}{ Female } & Incidence & $-2.688(-2.892,-2.484)$ & $<0.001$ & $<0.001$ & $<0.001$ \\
\hline & Mortality & $-3.531(-3.973,-3.087)$ & $<0.001$ & $<0.001$ & $<0.001$ \\
\hline
\end{tabular}

$\mathrm{Cl}$, confidence interval; IS, ischemic stroke; HS, hemorrhagic stroke.

mortality, the local drift values were almost below 0 in all age groups for both genders (Figure 2B,D,F).

Figure 3 shows the longitudinal age curves of type-specific stroke incidence and mortality by gender. The incidence of stroke continued on an upward trend for all age groups. It was noted that the fastest-growing age group was 65-85 years (Figure 3A,C,E). Stroke and its subtype mortality rates were also increasing for both genders, especially for those aged over 70 years (Figure 3B,D,F). Males had a higher stroke incidence and mortality than women across all age groups.

The estimated period RRs by gender are shown in Figure 4. Period RRs were found in similar declined declined patterns for type-specific stroke mortalities for both genders (Figure 4B,D,F), a more rapid decrease for females than males for the whole study period. Regarding incidence, period RRs showed a downward trend before 2005 and the RRs of females decreased faster than that of males before the RRs began to show an upward trend and RRs for males rose faster than females for stroke and IS (Figure 4A,E). Period RRs declined for both genders for HS incidence (Figure 4C) and it decreased quicker for females than males during the whole study period.

The estimated cohort RRs by gender are shown in Figure 5. Cohort effects for stroke incidence showed elevated trends peaking in the $1950 \mathrm{~s}$ and $1970 \mathrm{~s}$ for females and males respectively, before declining steadily for stroke (Figure 5A). For HS, cohort effect for incidence showed a monotonic decline trend (Figure 5C). For IS, trend of cohort effect for incidence is similar with stroke (Figure 5E). Cohort effects for mortality showed a monotonic declined trend. Cohort effects of HS decreased more than that for IS. The declined trends of the cohort RRs for mortality were more noticeable for females than that for males (Figure 5B,D,F).

The Wald test results for the APC analyses are presented in Table. For type-specific stroke incidence in both genders, significant differences in local drifts, cohort and period deviations indicated potential cohort and period effects on the observed temporal trends. For type-specific stroke mortality in both genders, significant differences in local drifts and cohort deviations indicated potential cohort effects on the observed temporal trends, though period effects could not be ruled out in the analysis of female data.

\section{Discussion}

In this study, we found that compared with the elevated trend of crude incidence and mortality rate, the ASIR showed a slightly increasing trend and the ASMR showed a decreasing trend. These trends can be explained from an age, period, and cohort prospective.

\section{Age Effects}

The rapid increase of the older population forms the foundation for stroke incidence and mortality. China had the largest population globally and has experienced a combined situation of accelerated aging and decreasing birth rate. Report of population prediction showed that between 2017 and 2050, the share of population who are aged $\geq 60$ years will increase from $16 \%$ to $35 \%$ in China. ${ }^{17,18}$

\section{Period Effects}

Period effects showed an opposite effect on the incidence for different types of stroke; a declined trend for HS, but an elevated trend for IS. These findings indicated different distribution of risks for the 2 specific types of stroke. During the past 3 decades, in China, the prevalence of risk factors for stroke has changed. Smoking had decreased from $37.6 \%$ to $28.15 \%$, hypertension increased from $13.6 \%$ to $23.2 \%$, obesity increased from $2.9 \%$ to $8.7 \%$, dyslipidemia increased from $18.6 \%$ to $39.91 \%$, and diabetes has increased from $2.51 \%$ to $10.9 \%$. Apart from the common risk factors such as smoking, IS was associated more closely with atherosclerosis risks such as diabetes, obesity and dyslipidemia than HS. The prevalence of atherosclerosis risks has been increasing since the 1990 s, which would result in an increase of IS. Meanwhile, serum cholesterol level was negatively associated with HS. The increase of dyslipidemia was accompanied by a decrease of HS inci- 
A

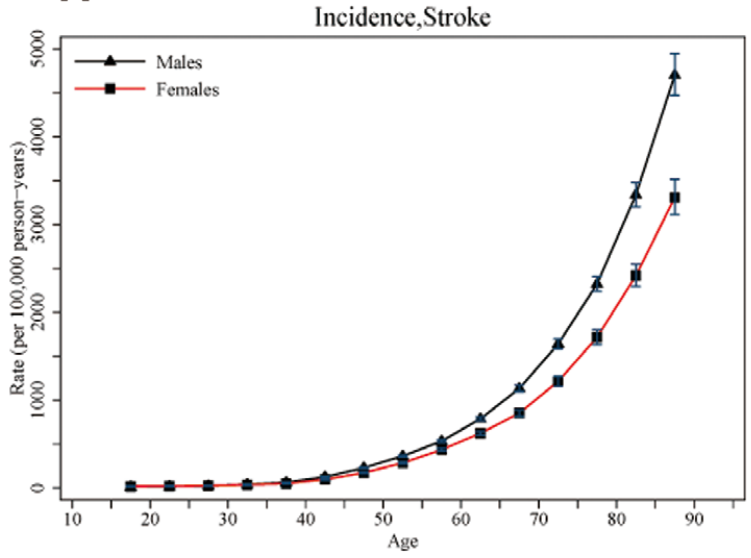

C

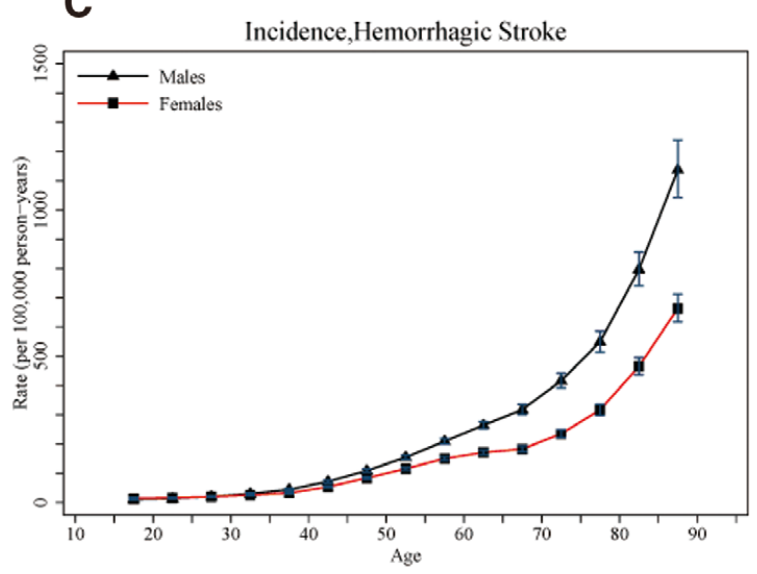

E

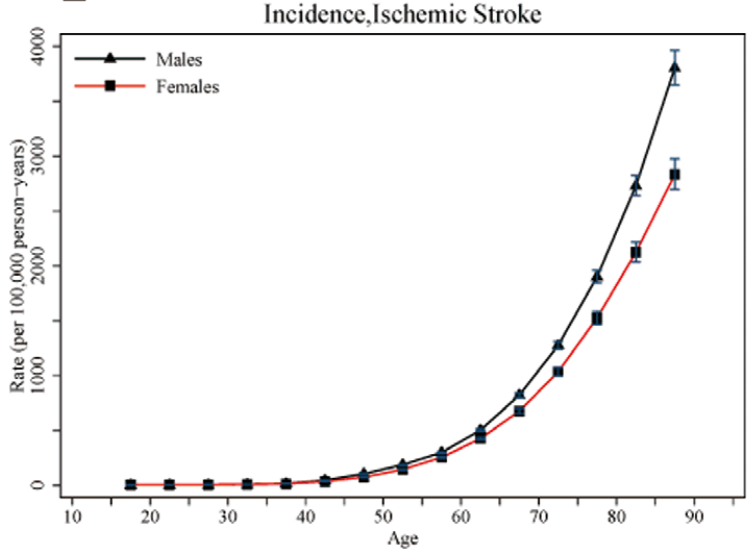

B

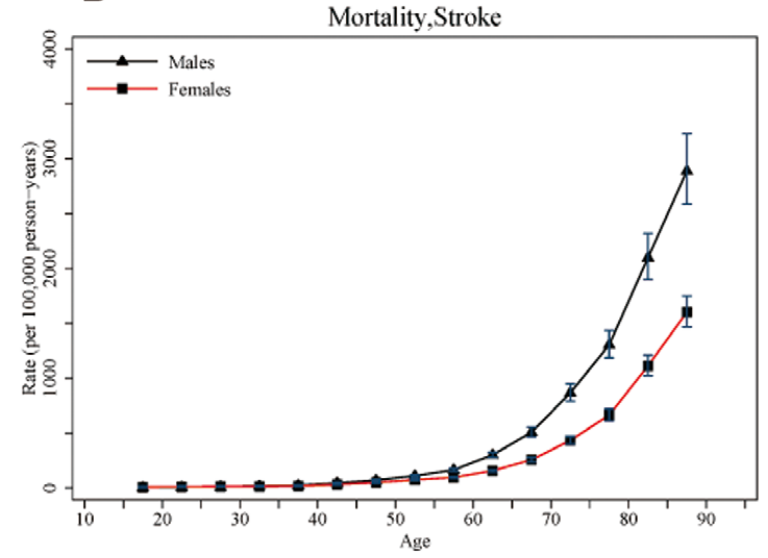

D

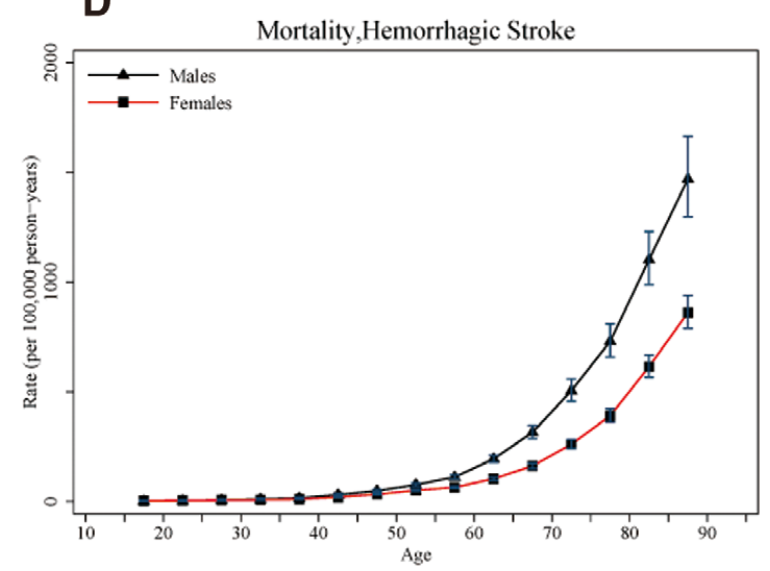

F

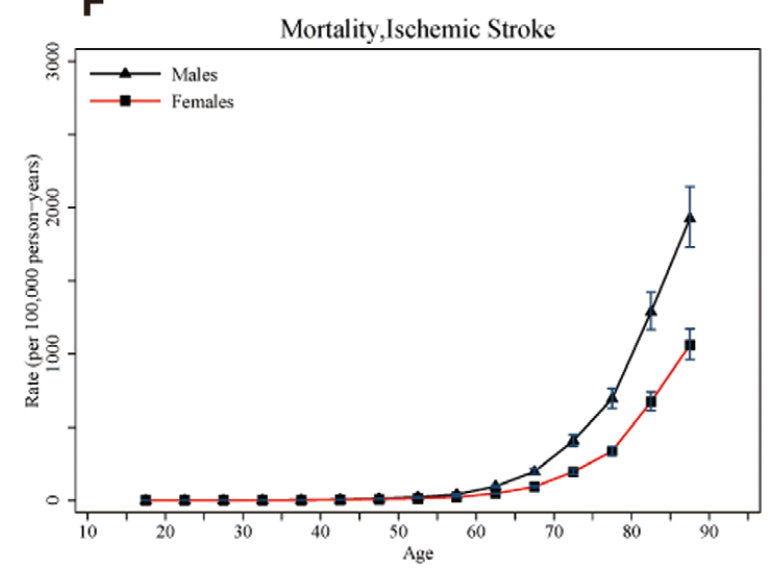

Figure 3. Longitudinal age curves of type-specific stroke incidence/mortality rates in China. Fitted longitudinal age-specific rates of type-specific stroke incidence/mortality rates (per 100,000 person-years) and the corresponding 95\% confidence intervals. (A) Stroke incidence rate. (B) Stroke mortality rate. (C) Hemorrhagic Stroke incidence rate. (D) Hemorrhagic Stroke mortality rate. (E) Ischemic Stroke incidence rate. (F) Ischemic Stroke mortality rate.

dence but there was an increase of IS incidence in the eastern Asian population. ${ }^{19}$

Improvement of medical care is the main reason of the monotonic declined association between period and stroke mortality. In China, the rapid socio-economic development improves stroke diagnosis and treatment, ${ }^{20,21}$ which decreases the fatality rate. As HS is the main sub-type of stroke mortality, HS is more beneficial than IS from the improved medical care. Meanwhile, the progress of urbanization and basic medical coverage promotes the availability, accessibility, and affordability of medical care in China. During 1990 to 2010, the proportion of the urban population increased from $26 \%$ to $50 \%$, and the coverage of basic medical services had achieved $90 \%$ in urban and 
A

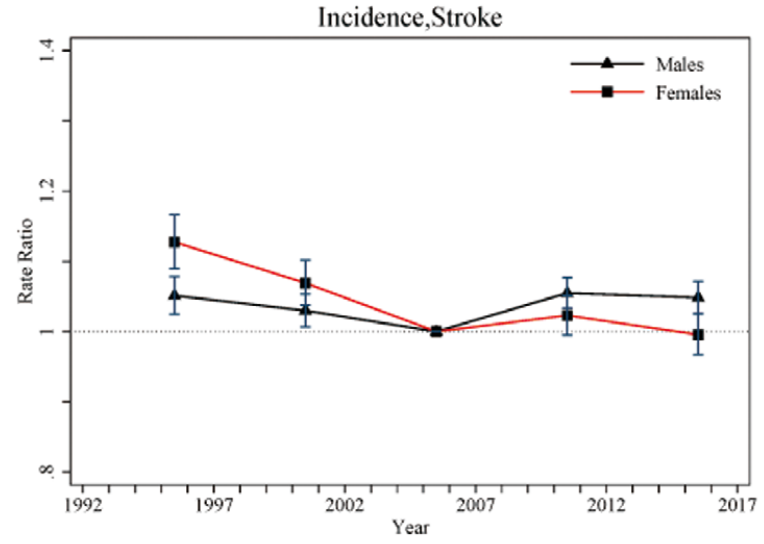

C

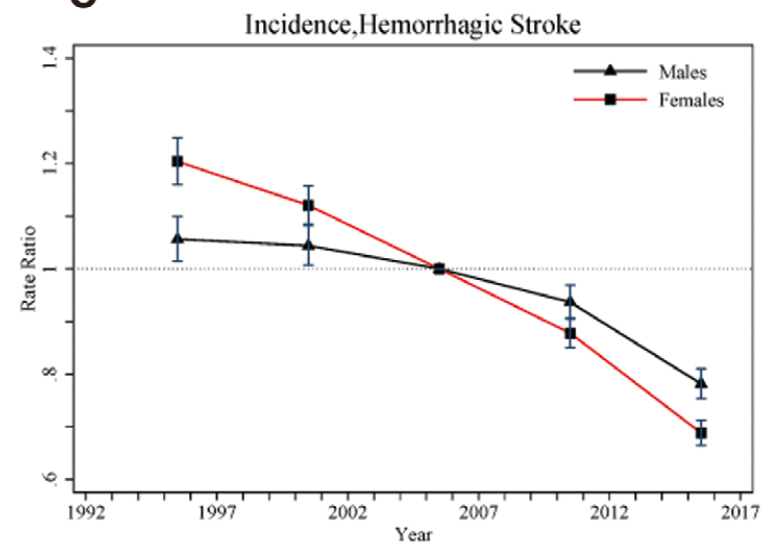

E

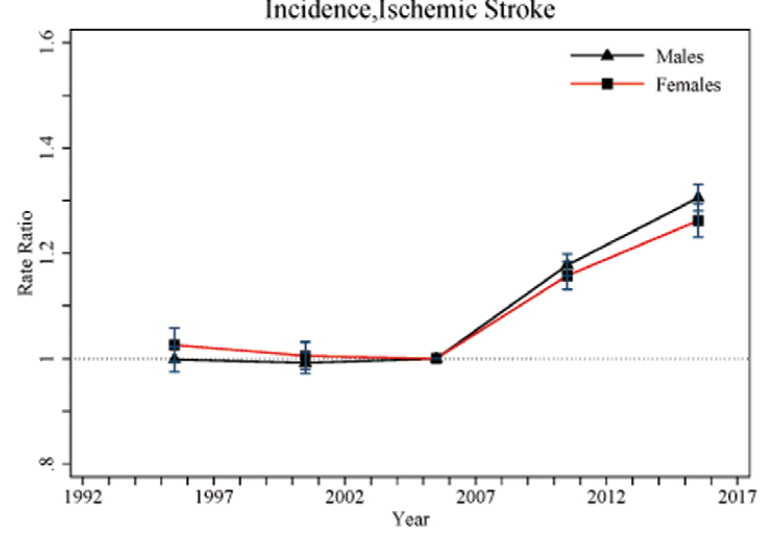

B

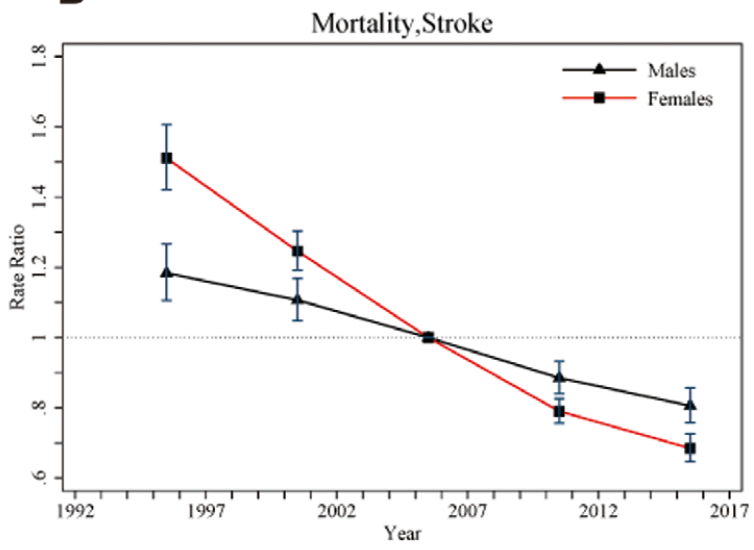

D

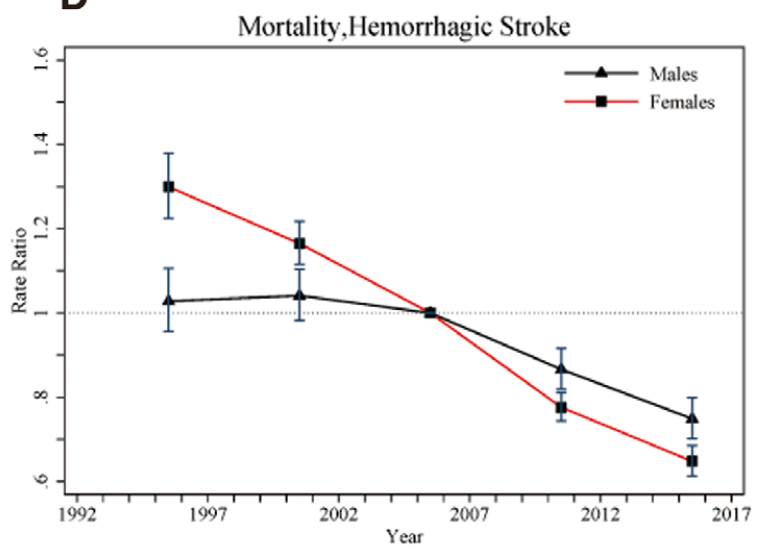

$\mathbf{F}$

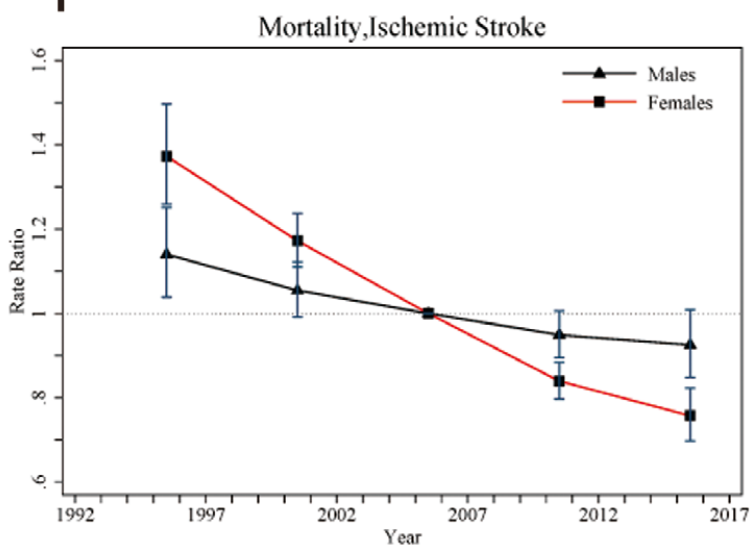

Figure 4. Period rate ratios (RRs) and the corresponding 95\% confidence intervals (95\% Cl) of type-specific stroke incidence/ mortality rates in China. The RRs of each period compared with the reference period (2003-2007) adjusted for age and non-linear cohort effects. (A) Period RRs and 95\% Cl of stroke incidence. (B) Period RRs and 95\% Cl stroke mortality. (C) Period RRs and 95\% $\mathrm{Cl}$ of hemorrhagic stroke incidence. (D) Period RRs and $95 \% \mathrm{Cl}$ of hemorrhagic stroke mortality. (E) Period RRs and $95 \% \mathrm{Cl}$ of ischemic stroke incidence. (F) Period RRs and $95 \% \mathrm{Cl}$ of ischemic stroke mortality.

\section{$95 \%$ in rural in $2013 .{ }^{22,23}$}

Cohort effects on stroke incidence showed an opposite trend for IS and HS, which could be explained by different type-specific risks. IS was associated with atherosclerosis more closely than HS. During the 1910s-1960s, China had experienced total wartime (Warlord dogfight [1917-1928], Anti-Japanese War and War of Liberation [1937-1949] and
Great Chinese Famine [1959-1961]). Long-term turbulence would bring high stress and induce negative emotions. ${ }^{24} \mathrm{It}$ can activate the sympathetic nervous system through the hypothalamic-pituitary-adrenal axis, ${ }^{25,26}$ which affects blood pressure, blood glucose and lipid metabolism. This association between chronic stress and cardio-cerebrovascular disease had been observed in refugees of the Croatian 
A

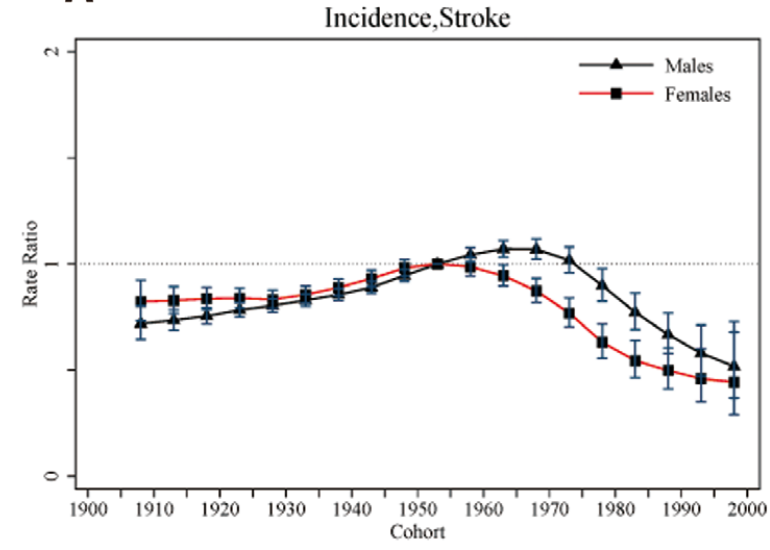

C

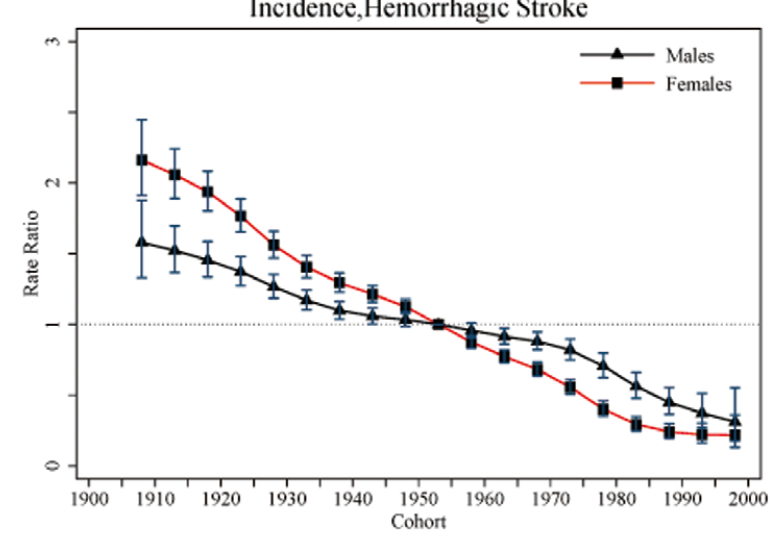

E

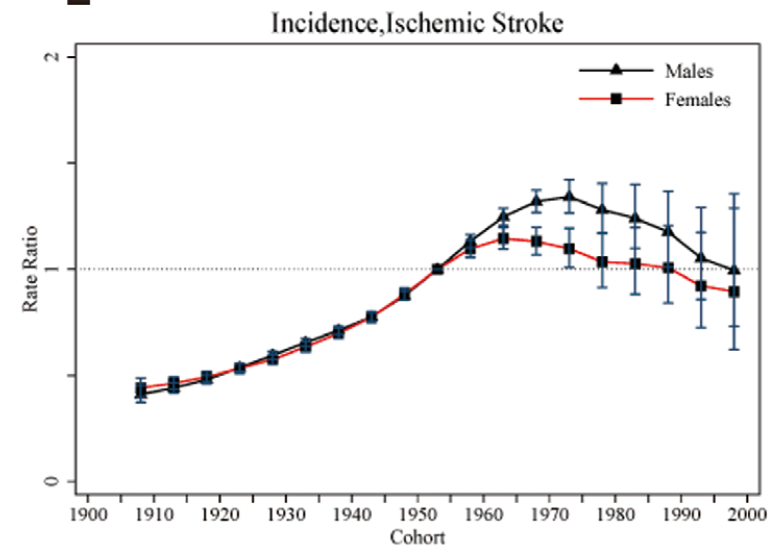

B

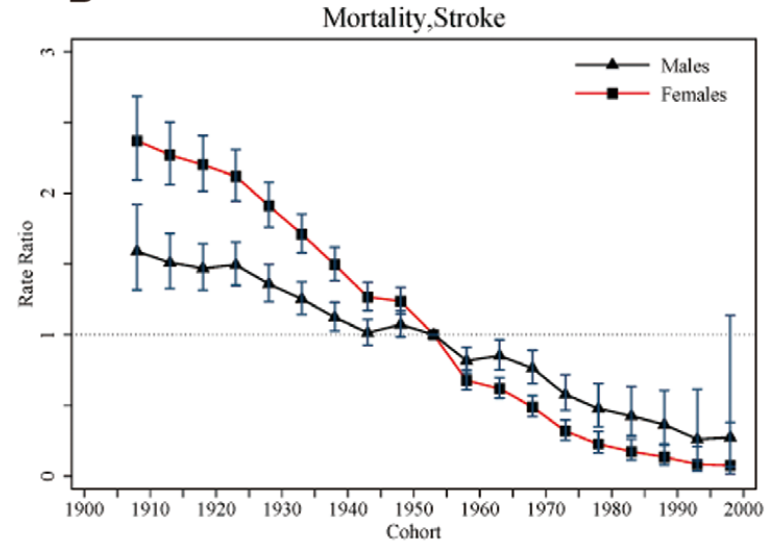

D

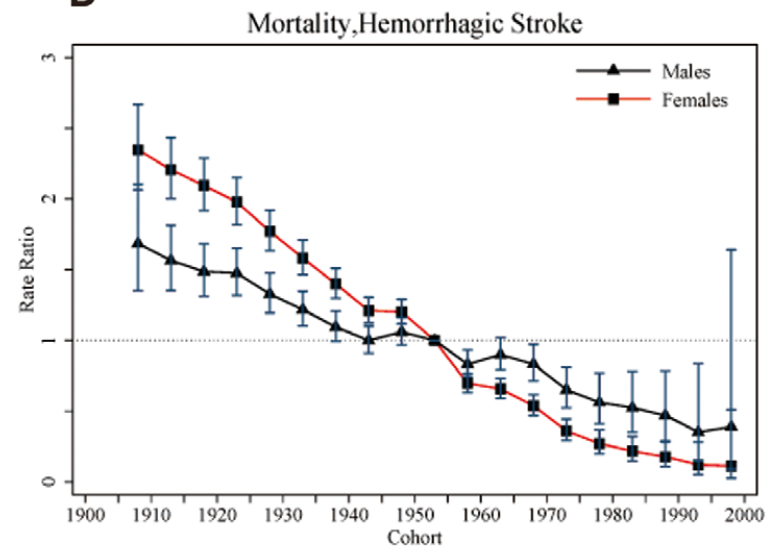

$\mathbf{F}$

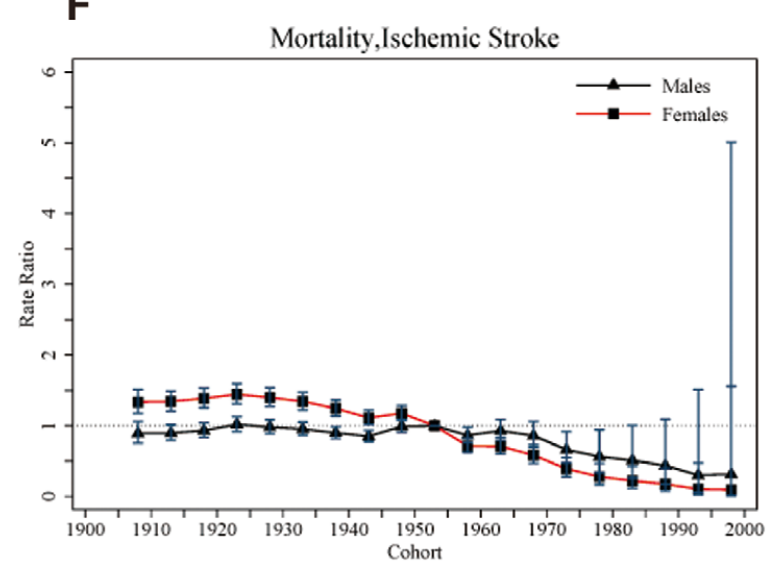

Figure 5. Cohort rate ratios (RRs) and the corresponding 95\% confidence intervals (95\% Cl) of type-specific stroke incidence/ mortality rates in China. The relative risk of each cohort compared with the reference cohort (1953 s) adjusted for age and non-linear period effects. (A) Cohort RRs and 95\% Cl of stroke incidence. (B) Cohort RRs and 95\% Cl stroke mortality. (C) Cohort RRs and $95 \% \mathrm{Cl}$ of hemorrhagic stroke incidence. (D) Cohort RRs and 95\% Cl of hemorrhagic stroke mortality. (E) Cohort RRs and $95 \% \mathrm{Cl}$ of ischemic stroke incidence. (F) Cohort RRs and $95 \% \mathrm{Cl}$ of ischemic stroke mortality.

War. ${ }^{26,27}$ Until the $1960 \mathrm{~s}-1970$ s, China had entered a period of economic development. The medical environment and technology had been improved. During the $1980 \mathrm{~s}-$ $2010 \mathrm{~s}$, rapid economic development resulted in the prevalence of metabolic syndrome. Thus, the accumulation of long-term stress during the turbulent period and the prevalence of atherosclerosis risks during the peaceful period may increase the risk of IS. When entered elder age, the cohort before 1910 may have a higher risk of IS than the cohort born after the $1970 \mathrm{~s}$. Meanwhile, the decline trend of the cohort effect on HS incidence could also be explained by the similar reason, as during the peace period, living and medical conditions had been improved for elderly which were born since $1910 \mathrm{~s}$. 
Cohort effects on mortality showed a declined trend for stroke, which was consistent with previous studies. ${ }^{4,8}$ These studies argued long-term improvement in nutrition in early life might be the main reason. However, as we mentioned before, China had experienced a long-term period of turbulent during the $1910 \mathrm{~s}-1960 \mathrm{~s},{ }^{24}$ and as a result, nutrition for children during their early years might not have seen improvement. A study in Japan found that the generation born during the war period experienced a lack of proper nutrition. ${ }^{7}$ Moreover, an APC study in China found different trends; for example, the cohort effect on stroke mortality remained steadily low during the war period, before increasing rapidly in urban areas, but remaining low in rural areas during peaceful times. ${ }^{28} \mathrm{~A}$ study in England confirmed that stroke mortality increased rapidly after the war because of the improvement in living conditions and the prevalence of an unhealthy lifestyle. ${ }^{6}$ In the present study, the declined cohort effects on stroke mortality might be due to the improved medical conditions, which reduced the number of fatality cases caused by stroke among generations born since the $1910 \mathrm{~s}$ when they entered older age during the peaceful period. ${ }^{29}$

There are several limitations in the present study. First, during the study period, the transition from ICD-9 to ICD-10 may influence statistical accuracy of stroke incidence and mortality. Yet, studies on cardio-cerebrovascular disease in the United States and China had shown that the transition of ICD only had minimal effect on stroke estimates. ${ }^{30,31}$ Second, despite many methods in Global Burden of Disease study estimations used to reduce bias, including misclassification corrections, incompleteness and redistribution of garbage codes, it might be difficult to avoid inaccuracy of data thoroughly; therefore, the results of the present study should be interpreted carefully.

\section{Conflicts of Interests}

The authors declare that they have no conflicts of interest.

\section{Grants}

This work was supported by the National Natural Science Foundation of China (Project number 71704131).

\section{References}

1. Peng $\mathrm{Q}, \mathrm{Li} \mathrm{HL}$, Wang $\mathrm{Y}, \mathrm{Lu} \mathrm{WL}$. Changing trend regarding the burden on cerebrovascular diseases between 1990 and 2016 in China. Zhonghua Liu Xing Bing Xue Za Zhi 2019; 40: 400-405 (in Chinese)

2. Zhao D, Liu J, Wang W, Zeng Z, Cheng J, Liu J, et al. Epidemiological transition of stroke in China: Twenty-one-year observational study from the Sino-MONICA-Beijing Project. Stroke 2008; 39: 1668-1674.

3. Wang W, Wang D, Liu H, Sun H, Jiang B, Ru X, et al. Trend of declining stroke mortality in China: Reasons and analysis. Stroke Vasc Neurol 2017; 2: 132-139.

4. Wang Z, Hu S, Sang S, Luo L, Yu C. Age-period-cohort analysis of stroke mortality in China: Data from the global burden of disease study 2013. Stroke 2017; 48: 271-275.

5. Ocana-Riola R, Blanco-Reina E, Navarro-Moreno E, MayoralCortes JM. Age-period-cohort effects on mortality from cerebrovascular disease in southern Spain. J Stroke Cerebrovasc Dis 2014; 23: 2274-2282.

6. Sutton CJ, Marsden J, Watkins CL, Leathley MJ, Dey P. Changing stroke mortality trends in middle-aged people: An age-periodcohort analysis of routine mortality data in persons aged 40 to 69 in England. J Epidemiol Community Health 2010; 64: 523 529.

7. Ma E, Takahashi H, Mizuno A, Okada M, Yamagishi K, Iso H. Stratified age-period-cohort analysis of stroke mortality in Japan, 1960 to 2000. J Stroke Cerebrovasc Dis 2007; 16: 91-102.

8. Li J, Li B, Zhang F, Sun Y. Urban and rural stroke mortality rates in China between 1988 and 2013: An age-period-cohort analysis. $J$ Int Med Res 2017; 45: 680-690.

9. Feigin VL, Krishnamurthi RV, Parmar P, Norrving B, Mensah GA, Bennett DA, et al. Update on the global burden of ischemic and hemorrhagic stroke in 1990-2013: The GBD 2013 Study. Neuroepidemiology 2015; 45: 161-176.

10. GBD 2017 Causes of Death Collaborators. Global, regional, and national age-sex-specific mortality for 282 causes of death in 195 countries and territories, 1980-2017: A systematic analysis for the Global Burden of Disease Study 2017. Lancet 2018; 392: $1736-1788$

11. Clayton D, Schifflers E. Models for temporal variation in cancer rates. I: Age-period and age-cohort models. Stat Med 1987; 6: $449-467$.

12. Clayton D, Schifflers E. Models for temporal variation in cancer rates. II: Age-period-cohort models. Stat Med 1987; 6: 469-481.

13. Holford TR. Understanding the effects of age, period, and cohort on incidence and mortality rates. Annu Rev Public Health 1991; 12: 425-457.

14. Holford TR. Analysing the temporal effects of age, period and cohort. Stat Methods Med Res 1992; 1: 317-337.

15. Rosenberg PS, Check DP, Anderson WF. A web tool for ageperiod-cohort analysis of cancer incidence and mortality rates. Cancer Epidemiol Biomarkers Prev 2014; 23: 2296-2302.

16. Holford TR. The estimation of age, period and cohort effects for vital rates. Biometrics 1983; 39: 311-324.

17. Liu T, Flothmann EJ. The new aging society: Demographic transition and its effects on old-age insurance and care of the elderly in China. Z Gerontol Geriatr 2013; 46: $465-475$ (in German).

18. Chen R, Xu P, Song P, Wang M, He J. China has faster pace than Japan in population aging in next 25 years. Biosci Trends 2019; 13: 287-291.

19. Blood pressure, cholesterol, and stroke in eastern Asia: Eastern Stroke and Coronary Heart Disease Collaborative Research Group. Lancet 1998; 352: 1801-1807.

20. Liu M, Wu B, Wang WZ, Lee LM, Zhang SH, Kong LZ. Stroke in China: Epidemiology, prevention, and management strategies. Lancet Neurol 2007; 6: 456-464.

21. Yu TS, Tse LA, Wong TW, Wong SI. Recent trends of stroke mortality in Hong Kong: Age, period, cohort analyses and the implications. Neuroepidemiology 2000; 19: 265-274.

22. Li X, Song J, Lin T, Dixon J, Zhang G, Ye H. Urbanization and health in China, thinking at the national, local and individual levels. Environ Health 2016; 15(Suppl 1): 32.

23. Li S. To establish essential medical security systems with Chinese characteristics. Acta Universitatis Medicinalis Nanjing 2014; 14: $260-265$.

24. Chen XG, PG W. Changes in China's society and the dynamic changes of national health. Chinese Journal of Population Science 2014; 63-73.

25. Everson-Rose SA, Roetker NS, Lutsey PL, Kershaw KN, Longstreth WT Jr, Sacco RL, et al. Chronic stress, depressive symptoms, anger, hostility, and risk of stroke and transient ischemic attack in the multi-ethnic study of atherosclerosis. Stroke 2014; 45: $2318-2323$.

26. Gallo LC, Roesch SC, Fortmann AL, Carnethon MR, Penedo FJ, Perreira K, et al. Associations of chronic stress burden, perceived stress, and traumatic stress with cardiovascular disease prevalence and risk factors in the Hispanic Community Health Study/Study of Latinos Sociocultural Ancillary Study. Psychosom Med 2014; 76: $468-475$.

27. Kadojić D, Demarin V, Barac B, Radanović B. 4-07-26 Influence of prolonged stress on stroke appearance. Journal of the Neurological Sciences 1997; 150: S219.

28. Wang YH. Trends in cerebrovascular disease mortality in China between 1990-2010. Chinese Journal of Disease Control \& Prevention 2013; 17: 7-11.

29. Lawlor DA, Smith GD, Leon DA, Sterne JA, Ebrahim S. Secular trends in mortality by stroke subtype in the 20th century: A retrospective analysis. Lancet 2002; 360: 1818-1823.

30. Qian Z, He Q, Lin HM, Kong L, Liao D, Gong J, et al. Exploring uncertainty of the change from ICD-9 to ICD-10 on acute mortality effects of air pollution. Environ Int 2008; 34: 248-253.

31. Panozzo CA, Woodworth TS, Welch EC, Huang TY, Her QL, Haynes K, et al. Early impact of the ICD-10-CM transition on selected health outcomes in 13 electronic health care databases in the United States. Pharmacoepidemiol Drug Saf 2018; 27: 839847. 